\title{
No evidence for a low-mass black hole in Swift J1753.5-0127
}

\author{
A. W. Shaw ${ }^{1 \star ~ P . ~ A . ~ C h a r l e s, ~}{ }^{1}$ J. Casares, ${ }^{2,3,4}$ and J. V. Hernández Santisteban ${ }^{1}$ \\ ${ }^{1}$ Department of Physics and Astronomy, University of Southampton, Southampton SO17 1BJ, UK \\ 2 Instituto de Astrofísica de Canarias, E-38205 La Laguna, Tenerife, Spain \\ ${ }^{3}$ Departamento de astrofísica, Univ. de La Laguna, E-38206 La Laguna, Tenerife, Spain \\ ${ }^{4}$ Department of Physics, Astrophysics, University of Oxford, Denys Wilkinson Building, Keble Road, Oxford OX1 3RH, UK
}

Accepted XXX. Received YYY; in original form ZZZ

\begin{abstract}
We present high-resolution, time-resolved optical spectroscopy of the black hole Xray transient Swift J1753.5-0127. Our optical spectra do not show features that we can associate with the companion star. However we do observe broad, double-peaked emission lines, typical of an accretion disc. We show that the mass of the compact object is likely $>7.4 \pm 1.2 M_{\odot}$, much higher than previous suggestions of a low-mass $\left(<5 M_{\odot}\right)$ black hole.
\end{abstract}

Key words: black hole physics - X-rays: binaries - X-rays: individual: Swift J1753.50127

\section{INTRODUCTION}

Galactic black hole X-ray transients (BHXRTs) are lowmass X-ray binaries (LMXBs) in which a black hole (BH) accretes material from a donor star via an accretion disc. Approximately $75 \%$ of all LMXB transients are believed to harbour a BH (McClintock \& Remillard 2006) and are characterised by long periods of quiescence (years to decades) followed by X-ray outbursts which can increase the luminosity by several orders of magnitude. BHXRTs have proven to be important in studying LMXBs, as in quiescence they provide the opportunity to study the donor itself, which is mostly impossible in luminous, persistent XRBs (Charles \& Coe 2006).

Using this technique of studying largely quiescent BHXRTs, and adding in the mass determinations in high mass X-ray binaries (HMXBs) there are now more than $\sim 20$ BH mass determinations (e.g. Casares \& Jonker 2014). The distribution of these masses has become the subject of intense scrutiny from both an observational and theoretical viewpoint as, when combined with the substantial number of accurate neutron star mass measurements, there appears to be a dearth of compact objects with masses in the range $2-5 M_{\odot}$ (Özel et al. 2010; Farr et al. 2011 and references therein). This has even been referred to as the "mass gap", and has prompted theoretical explanations that derive from the nature of the supernova mechanism that produced the $\mathrm{BHs}$, ranging from convective instabilities at the time of the explosion to 'failed' supernovae in a certain mass range of

^ E-mail: A.Shaw@soton.ac.uk red supergiants (Belczynski et al. 2012; Ugliano et al. 2012; Kochanek 2014). Consequently, there is considerable interest in either (a) accounting for this mass gap as a selection bias of some form (e.g. Farr et al. 2011; Kreidberg et al. 2012), or (b) finding objects with masses in or close to this range. Hitherto, the lowest mass BHXRT has been considered to be GRO J0422+32 $\left(\sim 5 M_{\odot}\right)$, but there has been a significant uncertainty in this value as a result of its poorly determined inclination (see Casares \& Jonker 2014 and references therein). The search has therefore been on for a low mass BHXRT with a high binary inclination, and hence a more accurate mass determination.

The subject of this work, Swift J1753.5-0127 (hereafter J1753.5) was discovered by the Swift Burst Alert Telescope (BAT; Barthelmy et al. 2005) in 2005 (Palmer et al. $2005)$ as a hard-spectrum ( $\gamma$-ray source) transient at a relatively high Galactic latitude $\left(+12^{\circ}\right)$. The source luminosity peaked within a week, at a flux of $\sim 200 \mathrm{mCrab}$, as observed by the Rossi X-Ray Timing Explorer (RXTE) All Sky Monitor (ASM; 2-12 keV) (Cadolle Bel et al. 2007). The source was also detected in the UV, with Swift's Ultraviolet/Optical Telescope (UVOT; Still et al. 2005), and in the radio with MERLIN (Fender et al. 2005). A $R \sim 15.8 \mathrm{mag}$ optical counterpart was identified by Halpern (2005), who noted that it had brightened by at least 5 magnitudes (as it is not visible in the Digitized Sky Survey; DSS), thereby establishing J1753.5 as an LMXB.

Subsequent time-resolved photometry of the optical counterpart (Zurita et al. 2008) revealed $R$-band modulations on a period of $3.24 \mathrm{~h}$, which were interpreted as a superhump period, $P_{\mathrm{sh}}$. Such periodicities are seen in high mass 
ratio (compact object/donor) CVs (the SU UMa systems) where it has been found by Patterson et al. (2005) that the period differential (defined as $\epsilon=\left(P_{\text {sh }}-P_{\text {orb }}\right) / P_{\text {orb }}$ ) is a function of the mass ratio. For the BH LMXBs $\epsilon$ is very small, and so the superhump period is $<2 \%$ longer than the system $P_{\text {orb }}$. The presence of superhumps in the optical light curves of J1753.5 are indicative of an accretion disc that is precessing due to perturbations by the companion and have been seen to occur in other BHXRTs such as XTE J1118+480 (Zurita et al. 2002).

More importantly for our study, the $R$-band modulations suggested that J1753.5 was a high inclination system (although not actually eclipsing or dipping) and hence held the potential for an accurate mass determination. This could be accomplished either (a) once it had returned to quiescence (although this would be challenging given its absence on the DSS), or (b) during outburst if it displayed fluorescence emission features on the surface of the donor whilst still X-ray active (e.g. Cornelisse et al. 2008).

Almost immediately after its peak the X-ray flux of J1753.5 started declining, but it then remained roughly constant at $\sim 20$ mCrab $(2-12 \mathrm{keV})$ for over 6 months rather than returning to quiescence as might have been expected for a typical BHXRT (McClintock \& Remillard 2006). The source has still not returned to quiescence $\sim 11$ years after its initial discovery, and has instead exhibited significant long-term ( $>400 \mathrm{~d})$ variability over the course of its prolonged 'outburst' (Shaw et al. 2013).

J1753.5 has remained as a persistent LMXB in a hard accretion state for the majority of this time, however it has experienced a number of short-term spectral softenings, characterised by an increase in the temperature of the inner accretion disc and simultaneous steepening of the power-law component in the X-ray spectrum (Yoshikawa et al. 2015). Investigation of the source during one such event with $R X T E$ revealed that it had transitioned to a hard intermediate accretion state. However, unlike the majority of BHXRTs, Swift J1753.5-0127 did not continue towards an accretion disc dominated soft state and instead returned to the hard state (Soleri et al. 2013). In early 2015, the source appeared to undergo another state transition when the Swift-BAT flux appeared to drop to its lowest levels since the source's discovery (Onodera et al. 2015). Subsequent follow-up with the Swift X-ray Telescope (XRT; Burrows et al. 2005), XMMNewton (Jansen et al. 2001) and the Nuclear Spectroscopic Telescope Array (NuSTAR; Harrison et al. 2013) revealed that J1753.5 had transitioned to one of the lowest luminosity soft states recorded in LMXBs (Shaw et al. 2015, 2016).

With a large $(\Delta R \sim 5 \mathrm{mag})$ optical increase at outburst, we would not expect to detect any spectroscopic signatures of the donor whilst it was active, due to the optical light being dominated by the accretion disc. Durant et al. (2009) confirmed this with spectroscopic observations revealing a smooth optical continuum and no evidence for features that could be associated with the donor. With no detectable fluorescence emission either, it had therefore not been possible to obtain any direct evidence of the compact object mass. However, INTEGRAL observations highlighted the presence of a hard power-law tail up to $\sim 600 \mathrm{keV}$, very typical of a black hole candidate (BHC) in the hard state (Cadolle Bel et al. 2007). Also, the power density spectrum from a pointed $R X T E$ observation revealed a $0.6 \mathrm{~Hz}$ quasi-periodic oscillation (QPO) with characteristics typical of BHCs (Morgan et al. 2005). QPOs have also been seen at $0.08 \mathrm{~Hz}$ in optical data (Durant et al. 2009) as well as in a number of X-ray observations after the initial outburst had declined (Ramadevi \& Seetha 2007; Cadolle Bel et al. 2007).

Remarkably, given the above summary of BHXRT properties, Neustroev et al. (2014, hereafter N14) have presented evidence that J1753.5 does contain a low mass $\left(<5 M_{\odot}\right)$ $\mathrm{BH}$, based on their discovery of narrow optical features (in both emission and absorption) which they associate with the donor, despite such features not being identifiable or visible in previous spectroscopic studies (Durant et al. 2009). Given the considerable potential importance of the identification of a high inclination, low-mass $\mathrm{BH}$ in the "mass gap", we therefore undertook a spectroscopic study of J1753.5, using significantly higher spectral resolution so as to investigate its properties in much greater detail, and at the very least attempt to confirm the low-mass BH candidacy of J1753.5. We therefore focussed on the donor's features reported by N14, and report here our inability to reproduce any of their results.

\section{OBSERVATIONS AND ANALYSIS}

J1753.5 was observed from 2015 June 14 21:39:36 UT to June 15 04:44:55 UT (MJD 57187.903-57188.198) with the Intermediate dispersion Spectroscopic and Imaging System (ISIS) on the 4.2-m William Herschel Telescope (WHT) at Observatorio del Roque de los Muchachos, La Palma, Spain. We obtained $25 \times 900$ s exposures covering a total spectral range

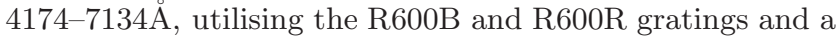
1 " slit in photometric conditions of good $(\sim 1$ ") seeing. We used IRAF (Tody 1986) to perform standard reduction techniques to achieve wavelength calibration, cosmic ray removal using the external task "lacos" (van Dokkum 2001) and extraction of the 1-dimensional spectra. $\mathrm{CuAr}+\mathrm{CuNe}$ comparision arcs were obtained to calibrate the wavelength scale, achieving a central dispersion of 0.43 and $0.49 \AA \mathrm{pixel}^{-1}$ in the blue and red arms, respectively. We obtain a spectral resolution of 1.55 and $1.66 \AA$ (FWHM) in the blue and red arms, respectively. We also observed the spectral type standard stars HR 4949 (M5III), HR 4986 (M0III), HR 4929 (K0III) and HR 4962 (K5III), whose spectra were reduced and extracted in the same way. We flux calibrated the averaged spectrum using the nearby flux standard star BD +33 2642 (Oke 1990). We note that the observations were performed when J1753.5 was in the soft state (Shaw et al. 2016).

Much of the analysis of the extracted spectra was performed using Tom Marsh's MOLLY software package. ${ }^{1}$ Computation of cross-correlation was performed separately for each arm of the spectrograph using the task xCOR, which computes the velocity shift of the target spectrum with respect to a template spectrum. We created 25 template spectra, each spectrum created by averaging all of the spectra of J1753.5 except for the target spectrum used for the

1 http://www2.warwick.ac.uk/fac/sci/physics/research/astro/people/marsh/s 
calculation of the cross-correlation. For example, we crosscorrelated spectrum 1 with a template created from the average of spectra $2-25$. This removes the possibility of a false positive correlation. We cut the spectra in the blue arm above $5500 \AA$ due to large-scale variations in the flatfields. The individual spectra and the template spectra were rebinned onto a uniform velocity scale in each arm and the continuum was subtracted by fitting a low-order spline to the data. Spectral features which may have affected the calculation of the cross-correlation function such as interstellar lines, $\mathrm{H}_{\alpha}$ and HeII $4686 \AA$ were masked out of the spectra and the templates before the calculation was performed. In the blue arm, cross-correlation was performed between 4174-

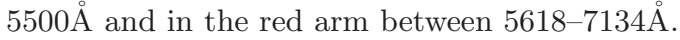

We also performed Doppler Tomography using the PYTHON implementation of Tom Marsh's DOPPLER package. ${ }^{2}$ We used the continuum subtracted spectra from each arm, trimmed around the lines of interest to compute the maps. To create the reconstructed trailed spectra from the resultant maps, we used the DOPPLER task COMDAT.

\section{RESULTS}

The flux-calibrated spectrum of J1753.5 is presented in Fig. 1 and exhibits strong double peaked HeII $4686 \AA$ and $\mathrm{H}_{\alpha}$ emission lines along with evidence of weak $\mathrm{H} \beta$ emission and He I $5876 \AA$. A number of diffuse interstellar bands (DIBs) are also present in the spectrum, most notably at $4428 \AA$ and $6283 \AA$ as well as the (resolved) interstellar $\mathrm{Na}$ $\mathrm{D}$ lines. We measure the equivalent widths (EWs) of the $\mathrm{Na} \mathrm{D}$ interstellar lines to be $0.58 \pm 0.03$ and $0.55 \pm 0.02$ $\AA$ for $\mathrm{Na}_{1}$ and $\mathrm{D}_{2}$ at 5889 and $5895 \AA$, respectively. This represents a decrease in EW from previous measurements (Durant et al. 2009) and such variability is indicative of some sodium being intrinsic to the system. The Fraunhofer B absorption features due to $\mathrm{O}_{2}$ are also apparent at $6867 \AA$. We find no absorption features in the averaged spectrum which might be identified with the secondary star.

\subsection{Companion star features}

N14 presented evidence of 'unidentified' narrow absorption and emission features in the spectrum of J1753.5, which showed a sinusoidal modulation over their claimed $2.85 \mathrm{~h}$ $P_{\text {orb }}$. They concluded that the features, the strongest of which were observed at 5193,5279 and $5356.1 \AA$, were associated with the companion star and therefore the observed modulation was due to its orbital motion.

In order to investigate this claim, we phase-folded our spectra on the $P_{\text {orb }}$ and ephemeris calculated by N14. We created a trailed spectrum of the region where such features were noted by N14 (5100-5400@), and present this in Fig. 2. Studying the trails in Fig. 2, we see no evidence for any of the moving features seen by N14 in our WHT spectra. We also note that there is also no evidence for such features when we phase-fold the data on the $P_{\text {orb }}$ determined by Zurita et al. (2008). To further examine the spectra for

${ }^{2}$ https://github.com/trmrsh/trm-doppler the presence of the features we also attempted to fit a number of gaussians centred on the wavelengths of the observed lines to each spectrum. This was a repeat of the analysis of N14, but found that the fits did not converge with sensible results, confirming our non-detection of these features.

\subsection{Cross-correlation}

Cross-correlation was performed using the MOLLY task XCOR as detailed above. The resultant cross-correlation function (CCF) spectra were then phase-folded on the $P_{\text {orb }}$ and ephemeris of N14, for comparison. We created trailed CCF spectra for each arm, which are presented in Fig. 3. The trailed spectra show no large deviations from $0 \mathrm{~km} \mathrm{~s}^{-1}$ and the sinusoidal modulation as seen by N14 is absent. We also note that there is no obvious modulation when the data are folded on $P_{\text {orb }}=3.24 \mathrm{~h}$ (Zurita et al. 2008).

We also cross-correlated the spectra of J1753.5 with four spectral type standards observed on the same night. We find no correlation with any of the spectral type templates, indicating that there are likely no features present in the spectrum of J1753.5 that can be associated with the range of late-type stars ( $\mathrm{K}$ and $\mathrm{M}$-type) most likely to be donors in this system.

\subsection{Disc emission lines}

The optical spectrum exhibits HeII $4686 \AA$ and $\mathrm{H}_{\alpha}$ emission lines. The lines are very broad and both exhibit a doublepeaked structure, very typical of emission features originating in an accretion disc (Smak 1981; Horne \& Marsh 1986), however they are weak $(\mathrm{EW} \sim 4 \AA$ for a double gaussian fit to each line). To examine the line profiles, first we fit a double gaussian to the emission lines in the phase-averaged spectrum, adopting a non-linear least-square approach. From this we can obtain an estimate of the rotational velocity at the outer rim of the disc, $v_{D} \sin i$, by measuring the observed peak-to-peak separation (Smak 1981; Warner 1995). Taking $\mathrm{H}_{\alpha}$, as this originates in the outermost regions of the accretion disc, we estimate $v_{D} \sin i=865 \pm 25 \mathrm{~km} \mathrm{~s}^{-1}$. For comparison, from the double-peaked HeII $4686 \AA$ emission we calculate $v_{D} \sin i=1115 \pm 29 \mathrm{~km} \mathrm{~s}^{-1}$, as expected since He II is formed closer in to the compact object.

The double-peak separation of the $\mathrm{H}_{\alpha}$ line profile can be used to estimate the radial velocity semi-amplitude, $K_{2}$ of the companion star in BHXRTs (Orosz et al. 1994; Orosz \& Bailyn 1995). In quiescent BHXRTs, the ratio $v_{D} / K_{2}$ has been shown to be $\simeq 1.1-1.25$. For J1753.5, we therefore find a very conservative lower limit of $K_{2}=$ $692 \pm 20 \mathrm{~km} \mathrm{~s}^{-1}$. It must be noted that this value is likely underestimated due to the source not yet being in quiescence. In outburst, the accretion disc expands to a radius larger than that in quiescence, therefore $v_{D}$ is smaller in outburst. $K_{2}$ can be used to constrain the mass of the compact object, $M_{1}$, by calculating the mass function:

$f\left(M_{1}\right)=\frac{P_{\text {orb }} K_{2}^{3}}{2 \pi G}=\frac{M_{1}^{3} \sin ^{3} i}{\left(M_{1}+M_{2}\right)^{2}}$

where $i$ is the inclination of the system and $M_{2}$ is the mass of the companion. In the case of J1753.5, which is a LMXB, then the mass function represents a firm lower limit to $M_{1}$. 


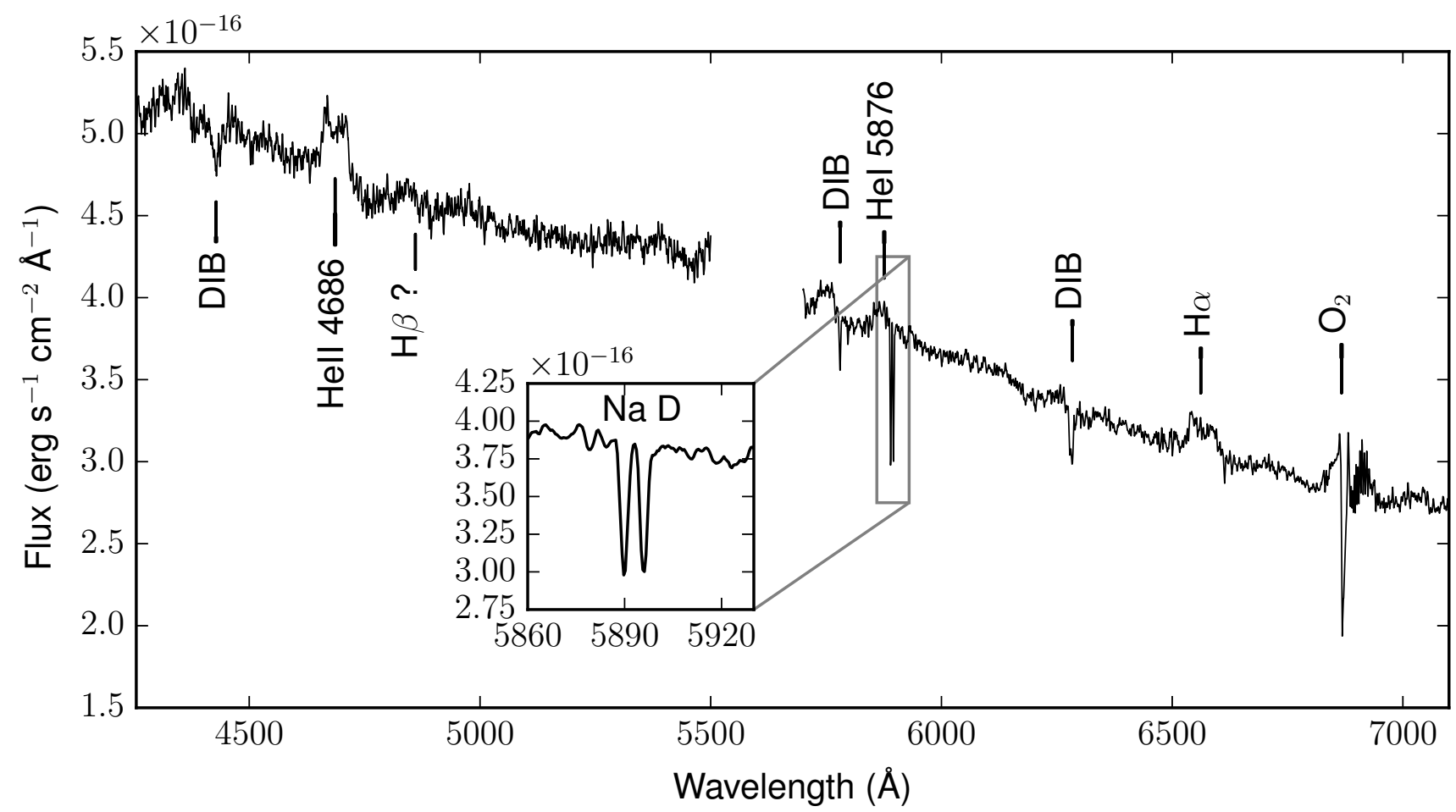

Figure 1. Flux calibrated averaged optical spectrum of J1753.5. Notable lines have been annotated. The inset shows a zoom of the Na D interstellar lines, highlighting that the two lines are resolved.

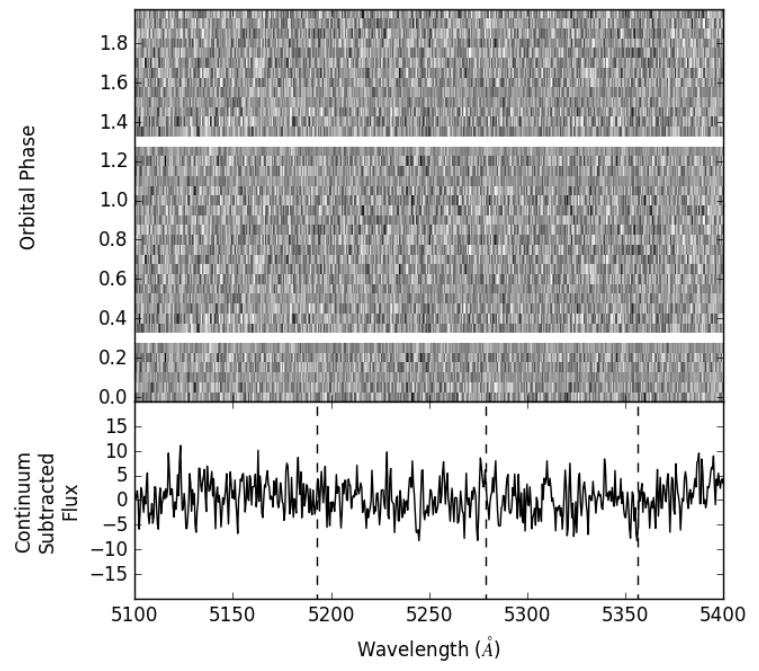

Figure 2. Top: The trailed spectrum of J1753.5 in the 5100$5400 \AA$ range, phase-folded on the $P_{\text {orb }}$ determined by N14. White indicates emission. Two cycles are shown for clarity. Bottom: The continuum subtracted, averaged spectrum of J1753.5. The dashed lines highlight the location of the narrow absorption and emission features seen by N14, which they associated with the secondary star.

Using $K_{2}=692 \pm 20 \mathrm{~km} \mathrm{~s}^{-1}$ and $P_{\text {orb }}=3.2443 \pm 0.0010 \mathrm{~h}$ (actually the superhump period only slightly larger than $P_{\text {orb }}$ Z Zurita et al. 2008); Zurita et al. 2008), we obtain $M_{1}>$ $4.6 \pm 0.3 M_{\odot}$. This again strongly indicates that the compact object in J1753.5 is a BH. The fact that this is a very conservative lower limit on the mass reinforces the case for a BH primary.

Recently, Casares (2015) discovered that the FWHM of a single gaussian fitted to the $\mathrm{H}_{\alpha}$ line profile is tightly correlated with $K_{2}=0.233(13) \mathrm{FWHM}$ in quiescent BHXRTs. As above, this relation is only valid for quiescence, but as the accretion disc expands during an outburst, the FWHM gets smaller, thus $\mathrm{FWHM}_{\text {(outburst) }}<\mathrm{FWHM}_{\text {(quiescence) }}$. We find FWHM $=3470 \pm 214 \mathrm{~km} \mathrm{~s}^{-1}$, which translates to $K_{2}>808 \pm 67 \mathrm{~km} \mathrm{~s}^{-1}$. When combined with $P_{\text {orb }}$ we find $M_{1}>7.4 \pm 1.2 M_{\odot}$. This again gives us a conservative lower limit on the mass of the compact object, higher than that calculated from the double peak separation.

Another way of constraining the mass of the compact object in J1753.5 is by scaling the FWHM of the $H_{\alpha}$ line with that of another system with well determined system parameters, such as XTE J1118+480. Since FWHM scales with $\sin i\left(\frac{M_{1}}{P_{\text {orb }}}\right)^{1 / 3}$ then:

$$
\begin{aligned}
& M_{1} \sin ^{3} i_{(\mathrm{J} 1753)}=M_{1} \sin ^{3} i_{(\mathrm{J} 1118)}\left(\frac{\mathrm{FWHM}_{(\mathrm{J} 1753)}}{\mathrm{FWHM}_{(\mathrm{J} 1118)}}\right)^{3} \\
& \times\left(\frac{P_{\mathrm{orb}(\mathrm{J} 1753)}}{P_{\mathrm{orb}(\mathrm{J} 1118)}}\right)
\end{aligned}
$$

Adopting for XTE J1118+480 the following parameters: $M_{1}=7.5 M_{\odot}, i=73^{\circ}$ (Khargharia et al. 2013), $P_{\text {orb }}=$ 0.170d (González Hernández et al. 2012) and FWHM=2850 $\mathrm{km} \mathrm{s}^{-1}$ (Casares 2015). Combining these values with our parameters for J1753.5, the scaling yields $M_{1} \sin ^{3} i_{(\mathrm{J} 1753)} \approx$ $9.4 M_{\odot}$ and even $\approx 8.3 M_{\odot}$ if $P_{\text {orb }}=2.85 \mathrm{~h}$ is assumed. Again, these values are lower limits because the FWHM has been 

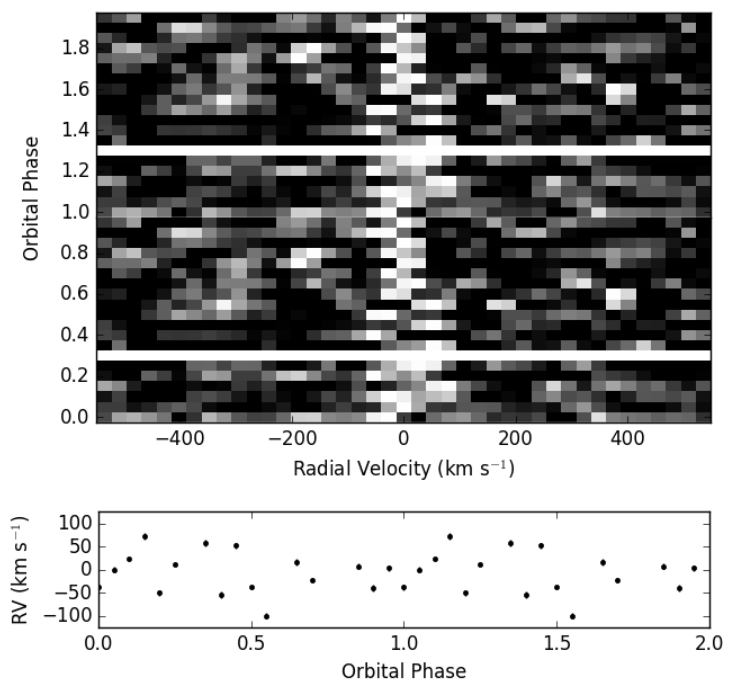
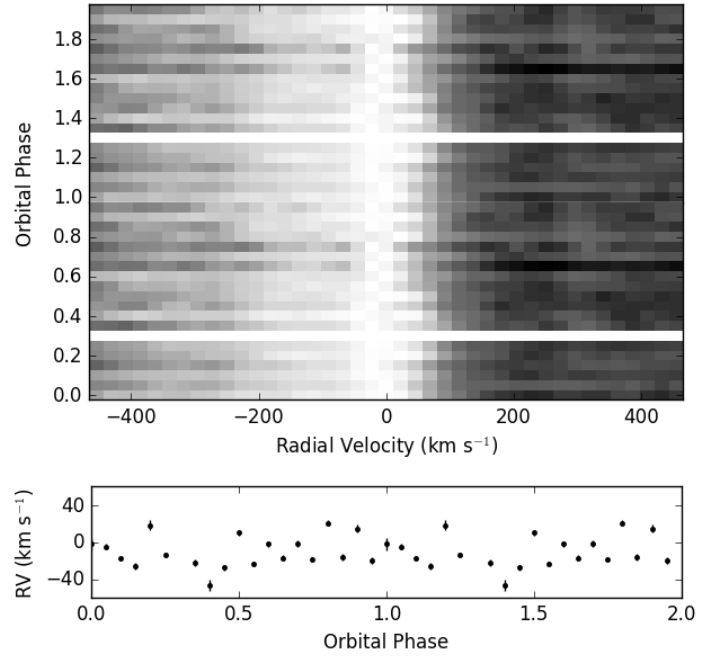

Figure 3. Left: Trailed, phase-folded CCF spectra from the blue arm. Right: Trailed, phase-folded CCF spectra from the red arm. Both datasets have been folded on the $P_{\text {orb }}$ determined by N14. The lower panels of each trailed spectrum show the peak radial velocity obtained from the CCFs for each phase. Two phases are shown for clarity.

measured in outburst and hence is an underestimate of the quiescent value. Therefore, the scaling of FWHM with XTE J1118+480 suggests that, in contrast with N14, the BH in $\mathrm{J} 1753.5$ is rather massive, even in the extreme case of an edge-on system.

We can also use the disc emission lines to investigate the motion of the compact object to the companion, i.e. $K_{1}$. To do this we cross-correlated the individual spectra with the phase-averaged spectrum, masking all but the He II and $\mathrm{H}_{\alpha}$ lines. However, the resultant CCFs were consistent with $0 \mathrm{~km} \mathrm{~s}^{-1}$ within $1 \sigma$ statistical uncertainties of $22 \mathrm{~km} \mathrm{~s}^{-1}$, indicating that we could not detect the orbital motion of the BH. This is contrary to N14, who claimed a value of $K_{1}=52 \pm 10 \mathrm{~km} \mathrm{~s}^{-1}$ by measuring the centroid of He II $4686 \AA$ emission feature, although N14 cautioned that the parameters obtained are plagued with systematic errors. We conclude that, though our spectra are higher resolution than those of N14, we cannot detect the orbital motion of the BH as we are limited by the signal-to-noise ratio of the individual spectra. Furthermore, the the overall emission line profile may not accurately trace the motion of the central star (see eg. Orosz et al. 1994).

\subsubsection{Doppler Tomography}

We can study the line profiles in more detail by examining the trailed spectra, which show variations of the blue and red peaks of the double-peaked line profiles. The orbital variation of the lines enables us to study the disc structure using the technique of Doppler tomography. We utilised the maximum entropy implementation of Doppler tomography using Tom Marsh's DOPPLER software package (see Marsh \& Horne 1988 for technical details). The resultant tomograms are presented in Fig. 4, where we have marked the Roche lobe of the compact object (dashed line) and the secondary (solid line) using the system parameters of
Zurita et al. (2008). The system parameters are not well known for J1753.5, so the plotted Roche lobes are estimates, nevertheless, they represent typical values for LMXB BH systems (see Casares \& Jonker 2014), which we believe to be more appropriate for J1753.5 (see section 4.3). The associated observed and reconstructed trailed spectra are presented in Fig. 5.

The tomograms show clearly the disc structure where the double peaked emission line profiles originate. The radii of the annuli are different for $\mathrm{He}$ II and $\mathrm{H}_{\alpha}$, with $\mathrm{H}_{\alpha}$ exhibiting smaller velocities - indicative of the smaller peak-to-peak separation in the line. The emission for both lines is roughly symmetric, showing none of the enhanced structure seen by N14. We constructed tomograms using the system parameters calculated by N14, also presented in Fig. 4. However, they do not exhibit the same 'clean' disc structure, instead showing four distinct regions of enhanced 'clumpy' emission rather than the uniform disc we see using the parameters from Zurita et al. (2008).

It is important to note that the tomograms computed with the system parameters of N14 in Fig. 4 show a disc constrained to emit only from an inner fraction of its Roche lobe. With ongoing mass transfer (for this very extended outburst) it is far more likely that emission extends out to velocities associated with the orbit of the donor. Such structure is then seen in the tomograms calculated using the parameters of Zurita et al. (2008) as is also clear in those of XTE J1118+480 (see Fig. 5 of Torres et al. 2002).

\section{DISCUSSION}

\subsection{Orbital Period}

N14 determined a period of $2.85 \mathrm{~h}$ by performing time-series analysis on both their spectroscopic and photometric data. The source is known to show strong modulations in its 

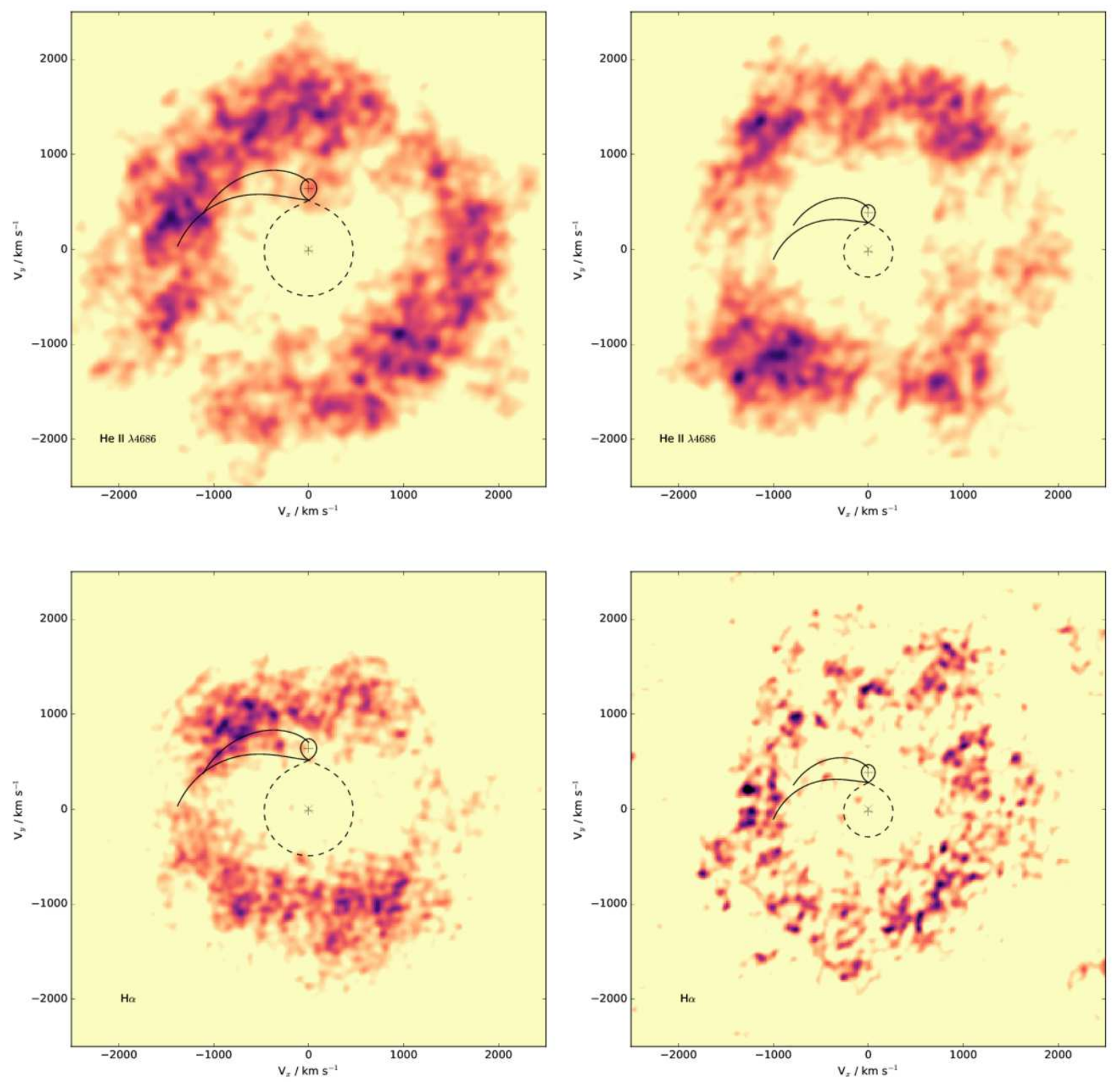

Figure 4. Doppler tomograms for the He II $4686 \AA$ (upper) and $\mathrm{H}_{\alpha}$ (lower) emission lines. The two left panels were constructed utilising the system parameters of Zurita et al. (2008), the two right panels were constructed using those of N14.

optical light curve (Zurita et al. 2008), but N14 also found similar variability to be present in their time-resolved spectroscopy. The Lomb-Scargle (Lomb 1976; Scargle 1982) periodograms for both their photometric and spectroscopic data show the strongest peaks at $\sim 8.4$ cycles $\mathrm{d}^{-1}$, leading to their proposed $P_{\text {orb }}=2.85 \pm 0.01 \mathrm{~h}$ to be the true orbital period. However, it is evident from their periodograms that there are a number of significant peaks close to the chosen frequency, one of which is the previously determined superhump period of $3.24 \mathrm{~h}$ (Zurita et al. 2008). It is clear that the timing analysis performed by N14 suffers from aliasing, which is most likely due to the low number of photometric observations obtained, many of which do not cover a complete orbital cycle. Zurita et al. (2008) obtained 20 photometric observations of $\sim 6 \mathrm{~h}$ per night, of significantly greater phase coverage. In addition, the variability of the nightly light curves is non-sinusoidal, meaning the phase-dispersion minimisation technique (PDM; Stellingwerf 1978) allowed a more accurate determination of the periodicities present than Lomb-Scargle time-series analysis. We therefore choose $P_{\text {orb }} \approx 3.24 \mathrm{~h}$ as the orbital period of J1753.5.

To further investigate this disagreement over which is the correct value of $P_{\text {orb }}$, we re-examined the light curves presented by Zurita et al. (2008). We selected $R$-band photometry from four consecutive nights (27-30 Jun 2007) of observations, in order to compare with the variability demonstrated in the spectroscopy of N14 A subset of the Zurita et al. (2008) light curves was chosen such that each night of photometry matched the observation duration of 

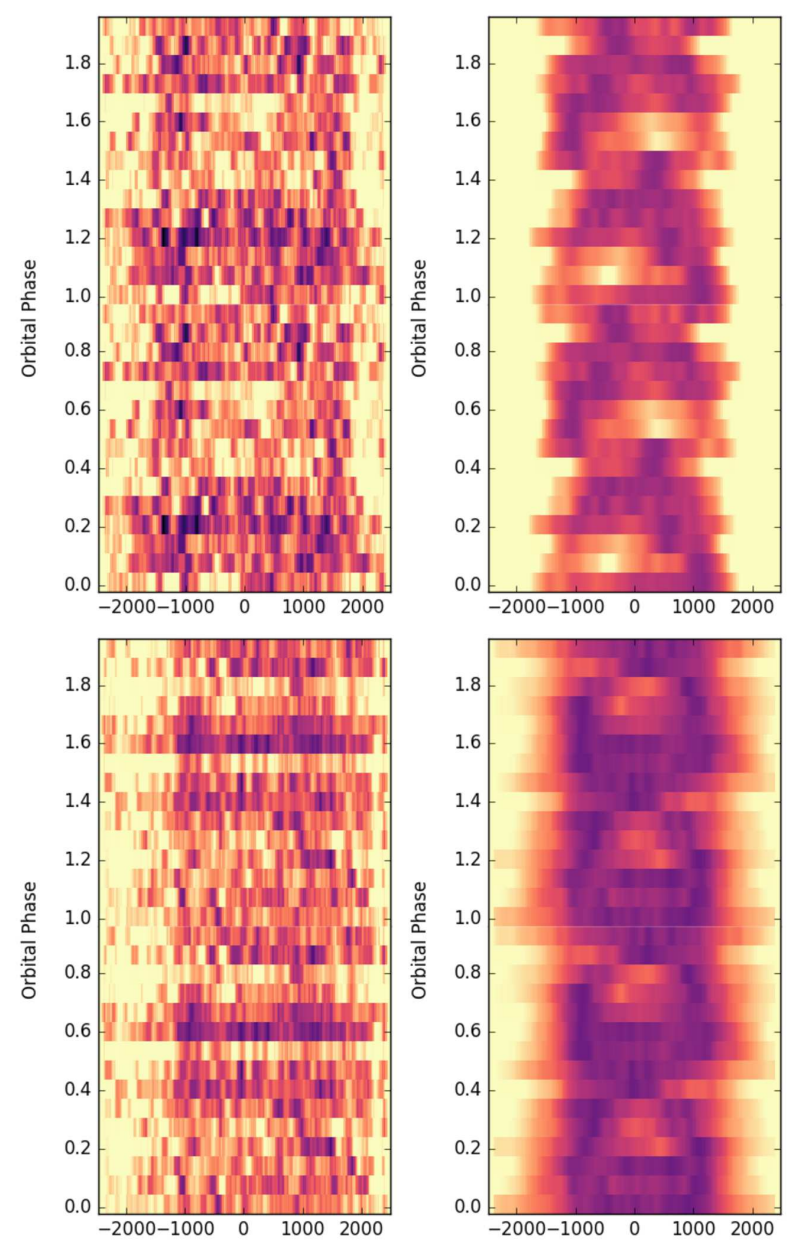

Figure 5. Observed and reconstructed trailed spectra for the $\mathrm{He}$ II $4686 \AA$ (upper) and $\mathrm{H}_{\alpha}$ (lower) emission lines. The trails were constructed using the system parameters of Zurita et al. (2008).

the corresponding spectroscopic observation of N14. For example, the 27 Jun 2007 observation was cut from $5.09 \mathrm{~h}$ to 1.97h to match the length of the Aug 062013 spectroscopic observation by N14. We then calculated the PDM and Lomb-Scargle periodograms, separately, for the photometry that matched the sampling of the N14 spectroscopy, and these are presented in Fig. 6. The upper two panels show that when the data has the same sampling as that of N14, aliasing becomes a serious problem.

The Lomb-Scargle periodogram (Fig. 6; middle panel) shows a very similar structure to N14's Fig. 4, with a number of peaks showing high power, including those corresponding to periods of $\approx 3.24 \mathrm{~h}$ and $\approx 2.85 \mathrm{~h}$. The morphology of the periodograms in Fig. 6 highlights the difficulty of disentangling which is the correct periodicity. The longer timebase and more extensive dataset of Zurita et al. (2008) removes this problem by including many more complete orbital cycles, with $\approx 3.24 \mathrm{~h}$ emerging as the most likely value of $P_{\text {orb }}$.

This is further illustrated in the bottom panel of Fig. 6, which shows the Lomb-Scargle periodograms of two simulated light curves. These have the same filtering applied to them as discussed above (i.e. the same sampling and duration as the N14 spectroscopy) but are

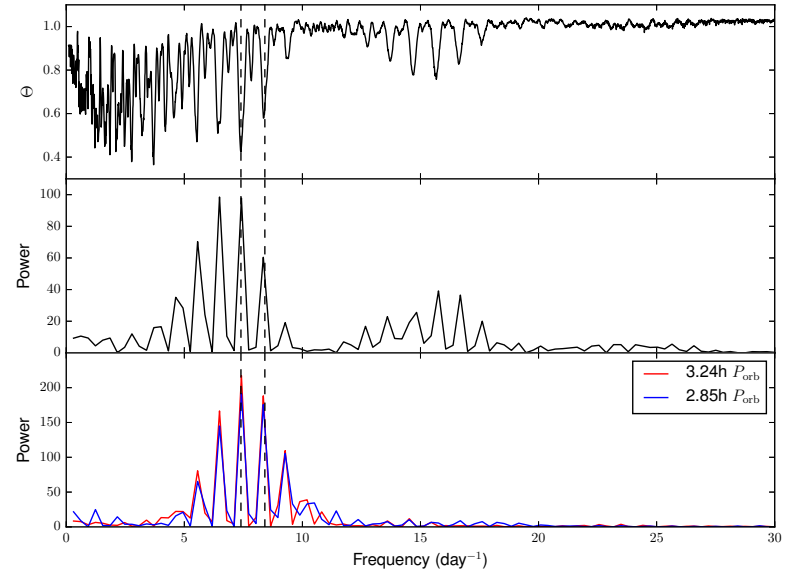

Figure 6. Top: PDM periodogram of the $R$-band nightly photometry from Zurita et al. (2008) which has been filtered to match the duration of the spectroscopic observations of N14 (see text for full description). Centre: Lomb-Scargle periodogram of the same photometry. Bottom: Lomb-Scargle periodograms of light curves created by simulating a sine curve with a period of $3.24 \mathrm{~h}$ (Red) and $2.85 \mathrm{~h}$ (Blue) using the same sampling as the filtered photometry in the upper two panels. The vertical dashed lines represent the two determined values of $P_{\text {orb }} ; 3.24 \mathrm{~h}$ (left) and $2.85 \mathrm{~h}$ (right).

simulated as a perfect sine curve with periodicities of $3.24 \mathrm{~h}$ and $2.85 \mathrm{~h}$. The periodograms of the simulated data are almost indistinguishable from one another, highlighting the problem of aliasing. Indeed, the periodogram of the $P_{\text {orb }}=2.85 \mathrm{~h}$ exhibits the highest peak at $3.24 \mathrm{~h}$, making it difficult to identify which is the true periodicity. Only with a longer data-set, such as that of Zurita et al. (2008), will the effects of aliasing be reduced and the true value of $P_{\text {orb }}$ become more apparent. This is seen in Fig. 7, which shows the Lomb-Scargle periodograms of two sine curves with periodicities of $3.24 \mathrm{~h}$ and $2.85 \mathrm{~h}$, created using the same sampling as the full light curves (i.e. 20 nights of photometry) presented by Zurita et al. (2008). It is clear from Fig. 7 that with a longer data-set, though aliasing is still apparent, it is more straightforward to determine which is the true period.

Contrary to N14, $P_{\text {orb }} \approx 3.24$ h puts J1753.5 outside the so-called 'period-gap' of binary systems in the range 2.15 $3.18 \mathrm{~h}$. In the more numerous accreting white dwarf (AWD) binaries, the well-determined period gap (Knigge et al. 2011) is explained by the switching off of the magnetic braking mechanism (which slows down the spin period of the companion star and hence reduces $P_{\text {orb}}$ ). This causes the donor star to shrink and mass-transfer stops due to the companion not filling its Roche lobe. It is not clear if there is the same period gap in BHXRTs as in AWDs (see e.g. King et al. 1996). Nevertheless, we find that the $P_{\text {orb }}$ of J1753.5 is close to the edge of this gap and thus may well be still filling its Roche lobe, which is evident from the persistent disc emission seen at optical and X-ray wavelengths. However, it is still unclear why the source has been persistent since its discovery as a transient in 2005 . 


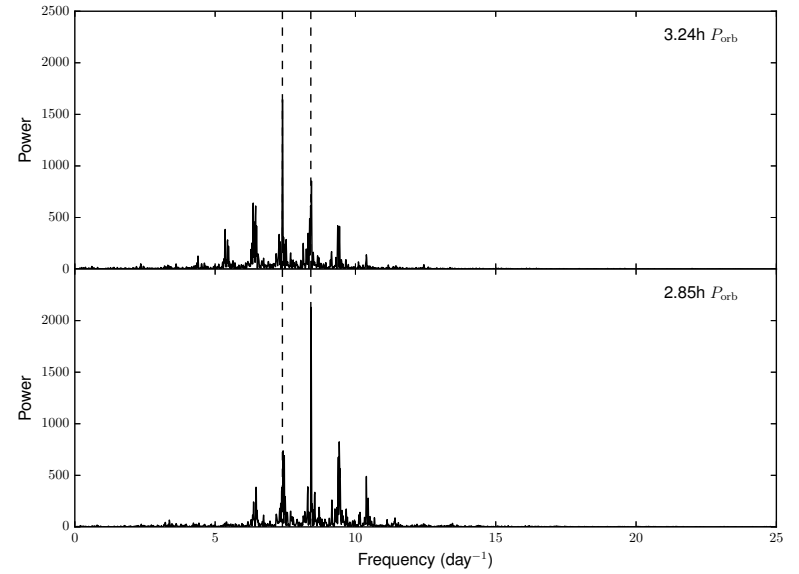

Figure 7. Lomb-Scargle periodogram of light curves created by simulating a sine curve with a period of $3.24 \mathrm{~h}(T o p)$ and $2.85 \mathrm{~h}$ (Bottom) using the same sampling as the light curves presented by Zurita et al. (2008). The vertical dashed lines represent the two proposed values of $P_{\text {orb }} ; 3.24 \mathrm{~h}$ and $2.85 \mathrm{~h}$ at frequencies of $\sim 7.4$ and $\sim 8.4$ day $^{-1}$.

\subsection{Companion Star Features}

We do not find the lack of features associated with the companion star to be surprising. J1753.5 has been a persistent X-ray source since its initial outburst in 2005 and is much brighter than the pre-outburst limit of the DSS $(R \lesssim 21)$, hence the donor must be fainter than this. Furthermore, the optical spectrum is very typical of that of an accretion disc, exhibiting very few features other than the broad, double-peaked HeII $4686 \AA$ and $\mathrm{H}_{\alpha}$ emission lines. The apparent companion features present in the optical spectrum of N14 remain unidentified, as (a) they are not at the wavelengths of any normal late-type stellar features and (b) we find no evidence for their presence in our higher resolution WHT spectrum (Fig. 2). Furthermore, the lack of any significant correlation with a wide range of late-type stellar template spectra suggests that any companion star features are washed out by the accretion disc.

However, it could be possible that the spectral templates we used do not accurately represent the spectral type of the companion star in J1753.5. For this reason we also cross-correlated each spectrum with templates created by averaging all the spectra, apart from the target spectrum of the cross-correlation. However, the CCFs showed none of the signals seen by N14 (Fig. 3). We therefore conclude that there are no spectral features associated with the companion present in our spectra of J1753.5.

The noise in our mean spectrum (Fig. 1) indicates that we would have been able to detect normal ( $\sim \mathrm{G}-\mathrm{K}$ star) absorption features had they been present at the $\sim 2 \%$ level with respect to the continuum (as with N14's spectrum). Since J1753 had $V \sim 17$ at the time of our observations (Neustroev et al. 2015) this implies that the donor must be fainter than $V \sim 21$, which is consistent with its non-detection in the DSS as noted earlier. We again point out that the so-called absorption features detected by N14 do not correspond with those of any normal late-type star and imply a donor that is brighter than this limit.

What could explain the apparently moving features seen by N14? The Boller and Chivens spectrograph on the $2.1 \mathrm{~m}$ telescope at the Observatorio Astronómico Nacional in Mexico was used to obtain the results presented by N14. They chose a wide slit width of 2.5 " despite the reported much better seeing conditions during the time of the observations. We point out that seeing variations and imperfect telescope pointing can lead to variations in the illumination profile of the source on the CCD. In particular, the 2.5" slit corresponds to $65 \mu \mathrm{m}$ at the spectrograph's plate scale of $38.4 " \mathrm{~mm}^{-1}$, which represents a wavelength spread of $8.1 \AA$ with their 400 lines $\mathrm{mm}^{-1}$ grating. If the seeing is much better than 2.5", then velocity shifts approaching $600 \mathrm{~km} \mathrm{~s}^{-1}$ could be induced by telescope tracking errors causing a shift of the stellar image within the slit.

However, this movement within the slit would only cause the variability seen if the N14 absorption features are real, yet we have been unable to confirm their existence. One explanation for this is to note that, when performing the cross-correlation, N14 appear to mask only the disc emission and night sky lines, which suggests that the interstellar absorption lines such as the DIBs and $\mathrm{Na} D$ were included in the calculation of the CCF. Therefore, telescope tracking errors could have given the impression of these features moving and could be responsible for the apparent RV variability that is seen by N14.

We also note that Fig 2. of N14 shows that the blue portion of the spectrum exhibits dramatic variability $(\sim 2 \times$ in flux) over the course of the observations, and this may also be a manifestation of the source drifting within the wide slit. Indeed, this interpretation is strongly supported by the stability of the B-band observations, which have only ever shown very low level variability $(<0.2 \mathrm{mag})$.

\subsection{Mass of the primary}

Using the recently derived $\operatorname{FWHM}\left(\mathrm{H}_{\alpha}\right)-K_{2}$ correlation (Casares 2015), we can obtain an independent estimate of the likely mass, $M_{1}$, of the primary. We find, conservatively, $M_{1}>7.4 \pm 1.2 M_{\odot}$, which is a strong indication that the primary is almost certainly a $\mathrm{BH}$, as also indicated by its spectral properties. N14 claim that it is a low mass $\mathrm{BH}\left(<5 M_{\odot}\right)$ based on their companion star radial velocity curve yielding a $K_{2}$ velocity of $\sim 380 \mathrm{~km} \mathrm{~s}^{-1}$, significantly lower than the estimates we present in this work. However, we are not able to replicate these results with our spectra. Therefore we suggest that the mass of the $\mathrm{BH}$ is much higher than previously suggested by N14 and hence there is no spectroscopic evidence for a low-mass BH in J1753.5.

N14 claim that J1753.5 lies in the so-called 'mass gap,' a gap in the distribution of known compact object masses in the range $2-5 M_{\odot}$ (Bailyn et al. 1998; Özel et al. 2010). The presence of sources in the mass gap may give an indication of the formation scenario of such systems, with BH masses in the range $2-5 M_{\odot}$ suggestive of a delayed supernova explosion scenario (Belczynski et al. 2012). However, with the mass estimate inferred from the $\mathrm{H}_{\alpha}$ FWHM we instead place J1753.5 well outside the mass gap, instead falling in the canonical BH mass distribution around $\sim 7 M_{\odot}$ (Özel et al. 2010). 


\section{CONCLUSIONS}

J1753.5 has now been active for $>10$ years, which has made it difficult to calculate the system's parameters using the normal methods applied to LMXB BH systems. We have obtained and analysed medium resolution spectroscopy in order to compare our results to those of N14, who claim a very low-mass primary and a short $(2.85 \mathrm{~h})$ orbital period. We find that we cannot replicate any of the results of N14, finding no spectroscopic evidence of the companion star. Instead, we derive a much higher compact object mass $\left(M_{1}>7.4 \pm 1.2 M_{\odot}\right)$, leaving no doubt that the source contains a $\mathrm{BH}$. We also find that the shorter $P_{\text {orb }}=2.85 \mathrm{~h}$ preferred by N14 is likely a result of aliasing, and our analysis supports a $P_{\text {orb }}$ of $3.24 \mathrm{~h}$ found by Zurita et al. (2008), which is more accurately interpreted as the (very fractionally longer) superhump period.

\section{ACKNOWLEDGEMENTS}

The authors would like to thank the anonymous referee for comments and suggestions which have helped improve this manuscript. AWS would like to thank Louise Wang for useful discussions regarding Doppler Tomography. JC acknowledges support by DGI of the Spanish Ministerio de Educación, Cultura y Deporte under grants AYA2013-42627 and PR2015-00397. Also to the Leverhulme Trust through grant VP2-2015-04. JHVS acknowledges financial support from CONACYT (Mexico) and the University of Southampton. This work made use of Tom Marsh's MOLLY and DOPPLER software packages. The authors would also like to thank Cristina Zurita Espinosa for providing us with her 2007 photometry of Swift J1753.5-0127.

\section{REFERENCES}

Bailyn C. D., Jain R. K., Coppi P., Orosz J. A., 1998, ApJ, 499,367

Barthelmy S. D., et al., 2005, Space Sci. Rev., 120, 143

Belczynski K., Wiktorowicz G., Fryer C. L., Holz D. E., Kalogera V., 2012, ApJ, 757, 91

Burrows D. N., et al., 2005, Space Sci. Rev., 120, 165

Cadolle Bel M., et al., 2007, ApJ, 659, 549

Casares J., 2015, ApJ, 808, 80

Casares J., Jonker P., 2014, Space Science Reviews, 183, 223

Charles P. A., Coe M. J., 2006, in Lewin W. H. G., van der Klis M., eds, Compact stellar X-ray sources. Cambridge Univ. Press, p. 215

Cornelisse R., Casares J., Muñoz-Darias T., Steeghs D., Charles P., Hynes R., O'Brien K., Barnes A., 2008, in Bandyopadhyay R. M., Wachter S., Gelino D., Gelino C. R., eds, AIP Conf. Ser. Vol. 1010, A Population Explosion: The Nature \& Evolution of X-ray Binaries in Diverse Environments. pp 148-152 (arXiv:0801.3367), doi:10.1063/1.2945024

Durant M., Gandhi P., Shahbaz T., Peralta H. H., Dhillon V. S., 2009, MNRAS, 392, 309

Farr W. M., Sravan N., Cantrell A., Kreidberg L., Bailyn C. D., Mandel I., Kalogera V., 2011, ApJ, 741, 103

Fender R., Garrington S., Muxlow T., 2005, ATel, 558, 1

González Hernández J. I., Rebolo R., Casares J., 2012, ApJ, 744, L25

Halpern J. P., 2005, ATel, 549, 1

Harrison F. A., et al., 2013, ApJ, 770, 103
Horne K., Marsh T. R., 1986, MNRAS, 218, 761

Jansen F., et al., 2001, A\&A, 365, L1

Khargharia J., Froning C. S., Robinson E. L., Gelino D. M., 2013, AJ, 145, 21

King A. R., Kolb U., Burderi L., 1996, ApJ, 464, L127

Knigge C., Baraffe I., Patterson J., 2011, ApJS, 194, 28

Kochanek C. S., 2014, MNRAS, 444, 2043

Kreidberg L., Bailyn C. D., Farr W. M., Kalogera V., 2012, ApJ, 757,36

Lomb N. R., 1976, Ap\&SS, 39, 447

Marsh T. R., Horne K., 1988, MNRAS, 235, 269

McClintock J. E., Remillard R. A., 2006, in Lewin W. H. G., van der Klis M., eds, Compact stellar X-ray sources. Cambridge Univ. Press, p. 157

Morgan E., Swank J., Markwardt C., Gehrels N., 2005, ATel, 550,1

Neustroev V. V., Veledina A., Poutanen J., Zharikov S. V., Tsygankov S. S., Sjoberg G., Kajava J. J. E., 2014, MNRAS, 445,2424

Neustroev V. V., et al., 2015, The Astronomer's Telegram, 7697 Oke J. B., 1990, AJ, 99, 1621

Onodera T., et al., 2015, The Astronomer's Telegram, 7196, 1

Orosz J. A., Bailyn C. D., 1995, ApJ, 446, L59

Orosz J. A., Bailyn C. D., Remillard R. A., McClintock J. E., Foltz C. B., 1994, ApJ, 436, 848

Özel F., Psaltis D., Narayan R., McClintock J. E., 2010, ApJ, 725,1918

Palmer D. M., Barthelmey S. D., Cummings J. R., Gehrels N., Krimm H. A., Markwardt C. B., Sakamoto T., Tueller J., 2005, ATel, 546, 1

Patterson J., et al., 2005, PASP, 117, 1204

Ramadevi M. C., Seetha S., 2007, MNRAS, 378, 182

Scargle J. D., 1982, ApJ, 263, 835

Shaw A. W., et al., 2013, MNRAS, 433, 740

Shaw A. W., Charles P. A., Gandhi P., Altamirano D., 2015, The Astronomer's Telegram, 7216, 1

Shaw A. W., et al., 2016, MNRAS, 458, 1636

Smak J., 1981, Acta Astron., 31, 395

Soleri P., et al., 2013, MNRAS, 429, 1244

Stellingwerf R. F., 1978, ApJ, 224, 953

Still M., Roming P., Brocksopp C., Markwardt C. B., 2005, ATel, 553,1

Tody D., 1986, in Crawford D. L., ed., Society of Photo-Optical Instrumentation Engineers (SPIE) Conference Series Vol. 627, Instrumentation in astronomy VI. p. 733

Torres M. A. P., et al., 2002, ApJ, 569, 423

Ugliano M., Janka H.-T., Marek A., Arcones A., 2012, ApJ, 757,69

Warner B., 1995, Camb. Astrophys. Ser., 28

Yoshikawa A., Yamada S., Nakahira S., Matsuoka M., Negoro H., Mihara T., Tamagawa T., 2015, PASJ, 67, 11

Zurita C., et al., 2002, MNRAS, 333, 791

Zurita C., Durant M., Torres M. A. P., Shahbaz T., Casares J., Steeghs D., 2008, ApJ, 681, 1458

van Dokkum P. G., 2001, PASP, 113, 1420

This paper has been typeset from a $\mathrm{T}_{\mathrm{EX}} / \mathrm{LAT}_{\mathrm{EX}}$ file prepared by the author. 